\title{
Acousto-Optics: introduction to the feature issue
}

\author{
Ting-Chung Poon, ${ }^{1, *}$ Chen S. Tsai, ${ }^{2}$ Vitaly B. Voloshinov, ${ }^{3}$ and Monish Chatterjee ${ }^{4}$ \\ 'Department of Electrical and Computer Engineering, Virginia Tech, Blacksburg, Virginia 24061, USA \\ ${ }^{2}$ Department of Electrical Engineering and Computer Science, University of California, Irvine, Mail Code ZOT 2625, \\ Irvine, California 92697, USA \\ ${ }^{3}$ Faculty of Physics, M. V. Lomonosov Moscow State University, 119992 Moscow, Russia \\ ${ }^{4}$ Department of Electrical and Computer Engineering, University of Dayton, 300 College Park, Dayton, Ohio 45469-0232, USA \\ *Corresponding author: tcpoon@vt.edu \\ Received 13 February 2009; accepted 13 February 2009; \\ posted 13 February 2009 (Doc. ID 107576); published 27 February 2009
}

\begin{abstract}
This Acousto-Optics feature celebrates the scientific careers of two remarkable scientists, Antoni Sliwinski and Adrian Korpel. The feature includes original papers based on a representative selection of topics that were presented at the Tenth Spring School on Acousto-Optics held in Poland in May 2008. (C) 2009 Optical Society of America

OCIS codes: $\quad 230.1040,070.1060$.
\end{abstract}

The years 2007 and 2008 marked milestones in the lives of two leading researchers and educators in the field of acousto-optics; Professors Adrian Korpel and Antoni Sliwinski turned 75 and 80, respectively. To commemorate these platinum and postplatinum milestones, the far-flung colleagues, collaborators, and students of these two accomplished scientists decided to put together a special issue of Applied Optics in their honor, dedicated to acousto-optics. With kind encouragement from that journal, it was decided that research papers submitted for the feature issue would contain a representative selection of original papers that were presented at the Tenth Spring School on Acousto-Optics in Sopot, Poland, in May 2008, as well as other appropriate papers that cover a range of topics.

Dr. Sliwinski, who has published articles on acousto-optics research for more than 40 years, was instrumental in developing the Spring School on Acousto-Optics and Applications in 1980, which brought together researchers on acousto-optics from across Europe, the United States, and elsewhere for close to 30 years. Dr. Korpel has made significant

0003-6935/09/07ACO1-02\$15.00/0

(C) 2009 Optical Society of America contributions to the understanding of acousto-optics by means of his development of the multiple planewave scattering formalism, the Feynman diagram approach, and other analytical models for weak and strong interactions for planar and curved wavefronts. Along the way, they have both extensively published papers, monographs, and books, several of which are regarded as classics in the field. As university educators, they have also guided and inspired many students and researchers to pursue careers around acousto-optics and other areas of opticsrelated research.

This feature issue contains a total of 23 papers, including one by Dr. Korpel and another by Dr. Sliwinsky co-authored with Piotr Kwiek. All the papers underwent substantial peer review under the guidelines of Applied Optics. In his short article, Dr. Korpel gives a riveting account of his personal discovery of acousto-optics and how the spirit of delight (childhood "curiosity and playfulness," as he describes it) guided his work in this and other fields, something he continues, postretirement, in activities as diverse as writing poems, essays, and short stories. In their article, Drs. Kwiek and Sliwinski lay out in revealing detail the international research collaboration that has resulted from the Spring Schools on Acousto-Optics held in Poland since 1980. The 
essay profiles the scientific interactions, aided by historic photographs and figures.

The twenty-one research papers can be broadly divided into two groups: sixteen that directly describe acousto-optic interactions and associated applications, when present, and five that primarily describe measurements or characterizations based on the use of light and sound. Four of the papers based on acousto-optic interactions deal with acousto-optic tunable filters (AOTFs). Kastelik et al. describe a tunable filter based on successive anisotropic interactions in paratellurite. Dobrolenskiy et al. investigate the effect of nonreciprocal behavior on the parameters of tunable filters and other devices at or above $1 \mathrm{GHz}$. Tseng and Tarn propose a polarization mode dispersion monitoring scheme for erbiumdoped fiber amplifiers using an AOTF. Gupta presents experimental results for AOTFs made from mercurous halide crystals suitable for operation in the UV to the longwave infrared (LWIR) range with reasonable performance characteristics. Seven papers deal with the investigation of fundamental acousto-optic interactions. Voloshinov and Polikarpova investigate unusual propagation and reflection of acoustic waves in anisotropic materials such as paratellurite using acousto-optic interactions. Grulkowski et al. consider the interaction of airborne cylindrical ultrasound with laser light inside $\mathrm{He}-\mathrm{Ne}$ and argon-ion laser cavities. Bukowski analyzes the Raman-Nath interaction of a Gaussian optical beam with the nonstationary temperature field of a onedimensional thermal wave. Zyuryukin and Yulaev apply a vector diagram concept to the collinear, anisotropic interaction of harmonically modulated light with a standing acoustic wave. Balakshy and Mantsevich examine the diffraction characteristics of divergent optical beams interacting collinearly in calcium molybdate and paratellurite. Pieper et al. derive exact analytical solutions for four-order Bragg diffraction under arbitrary initial conditions. $\mathrm{Ng}$ et al. use numerical analysis based on optical diffraction to characterize liquid surface acoustic waves effectively. Five papers emphasize applica- tions of acousto-optics. Balakshy and Kostyuk describe acousto-optic image processing in some detail and present a new method for visualizing phase objects. Solodov et al. develop a theory for the interaction between a narrow laser beam and acoustic waves in air using concepts of reflection vibrometry. Petkovsek et al. use photoelastically induced birefringence in lithium niobate via mechanical oscillations that are due to an applied voltage to examine the propagation of polarized light. Molchanov et al. analyze dispersive AOTFs based on quasicollinear interactions in paratellurite and conduct experiments for femtosecond laser pulse shaping. Antonov et al. investigate multifunctional switching (spatial multiplexing) of light beams across fiberoptic channels using light diffraction by multifrequency sound waves and demonstrate improvements in number of channels, switching time, and cross talk. The remaining five papers include one by Guo et al. that demonstrates a laser photothermal instrumentation technique useful in measuring steel hardness online. Egerev et al. discuss a physical model suitable for describing optical to acoustical conversion during irradiation of metal nanoparticles with laser pulses by means of optical-plasmon interactions. Bodzenta et al. examine thermal diffusivity of three doped single crystals used in the solidstate laser application of a thermal wave method. Skrodzka et al. examine modal frequencies of violins using both mechanical and optical investigation methods. Finally, Kazmierczak-Balata et al. determine the thermal conductivities of thin films on semiconductor substrates using photothermal experiments.

This feature issue benefited from the outstanding coordination efforts by Keith Jackson and Joseph Richardson, who ensured that the feature editors were continually engaged in the review process to ensure timely completion of the reviews. Thanks are also due to Lucille Halberstadt for the cover page design and to all the contributors whose participation made this issue possible. 